\title{
Inspecting the electronic architecture and semiconducting properties of a rosette-like supramolecular columnar liquid crystal
}

\author{
Beatriz Feringán,[a] César L. Folcia,[b] Roberto Termine,[c] Attilio Golemme,[c] José M. \\ Granadino-Roldán,[d] Amparo Navarro, *[d] José Luis Serrano,[e] Raquel Giménez, ${ }^{*}[a]$ Teresa \\ Sierra*[a]
}

[a] Dr. B. Feringán, Dr. R. Giménez, Dr. T. Sierra

Departamento de Química Orgánica. Facultad de Ciencias. Instituto de Ciencia de Materiales de Aragón (ICMA). Universidad de Zaragoza-CSIC. 50009 Zaragoza, Spain. E-mail:

rgimenez@unizar.es, tsierra@unizar.es

[b] Prof. C. L. Folcia

Departamento de Física de la Materia Condensada, Facultad de Ciencia y Tecnología, UPV/EHU, 48080 Bilbao, Spain

[c] Dr. R. Termine, Dr. A. Golemme

LASCAMM CR-INSTM, CNR-NANOTEC Lab LiCryL, Dipartimento di Fisica, Università della

Calabria, 87036 Rende, Italy

[d] Dr. A. Navarro, Dr. J. M. Granadino-Roldán

Departamento de Química Física y Analítica. Facultad de Ciencias Experimentales. Universidad de Jaén, Campus Las Lagunillas, 23071 Jaén, Spain. anavarro@ujaen.es

[e] Prof. J. L. Serrano

Departamento de Química Orgánica. Facultad de Ciencias. Instituto de Nanociencia de Aragón (INA). Universidad de Zaragoza. 50018 Zaragoza, Spain

Electronic Supplementary Information (ESI) available: [synthesis, characterization techniques, POM and DSC, computational details]. See DOI: 10.1039/x0xx00000x

\begin{abstract}
Computational and experimental studies unravel the structural and electronic properties of a novel supramolecular liquid crystal built through a hierarchical assembly process resulting in an $\mathrm{H}$-bonded melamine rosette decorated with peripheral triphenylenes. The six-fold symmetry of the mesogen facilitates the formation of a highly organized hexagonal columnar mesophase stable at room temperature. X-ray diffraction and electron density maps confirm additional intra- and intercolumn segregation of functional subunits, and this paves the way for 1D charge transport. Indeed, hole mobilily has been measured and found to be higher than for related mesogens. DFT calculations of HOMO and LUMO levels and parameters such as reorganization energy and transfer integral of the rosette structure have been achieved, and not only validate the columnar organization but also establish the way it translates into a favorable electronic architecture and molecular orbital interactions to promote charge carrier mobility.
\end{abstract}


The formation of columnar mesophases through the hierarchical assembly of small building blocks permits strict control of intermolecular interactions, thus aiding the optimization of material properties. In this respect, complementary non-covalent interactions, together with the assistance of nanosegregation processes of incompatible parts, has allowed the development of functional columnar liquid crystals with properties of technological interest, including ferroelectricity, selective recognition processes, ion-conduction, and chirality, amongst others.1

In the case of organic semiconductors, tuning of the position and orientation of molecular units and the electronic distribution is important in order to optimize the overlap between molecular orbitals and hence the macroscopic manifestation of charge carrier mobility. In practice, nanosegregation of electroactive units in independent regions within columnar architectures has been described as a strategy to improve semiconducting properties in organic materials. 2

In this respect, we decided to exploit a combined columnar/supramolecular strategy, 3 by exploring, from experimental and theoretical points of view, a unique structure consisting of a rosette with six-fold symmetry formed by $\mathrm{H}$-bonding of six melamines and six carboxylic acids. This rosette structure, which has melamine-melamine and melamine-acid $\mathrm{H}$-bonds, was reported to form highly organized columnar mesophases.4 However, the achievement of this type of hierarchical organization with semiconducting units to enforce a novel organization with a high degree of order and 1D nanosegregation that would permit the appropriate electronic distribution for charge transport has not been approached before. In particular, as shown in Figure 1, in the work reported here the melamine (M)-derived rosette was decorated with six molecules of a triphenylene-containing acid (TPC10), which places electron-donor blocks around an electrondeficient macrocycle of triazine rings to form the C6 complex M6-(TPC10)6.

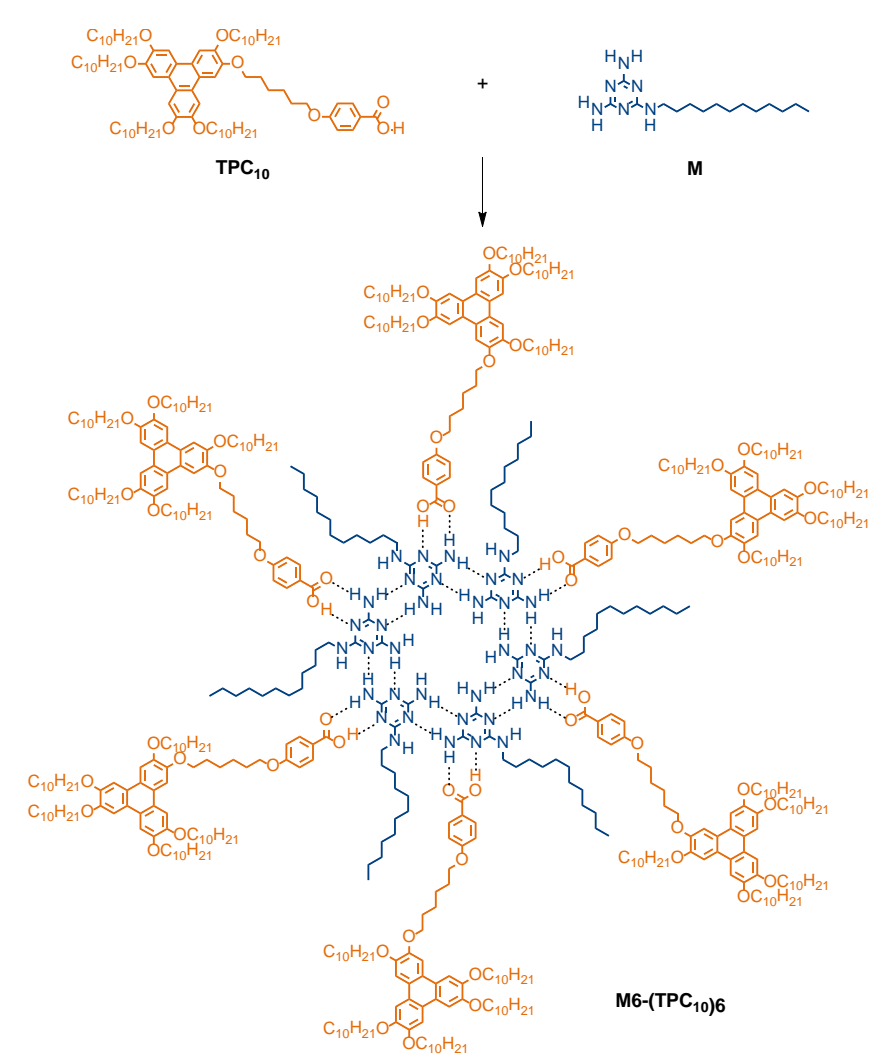

Figure 1. Chemical structure of the rosette complex M6-(TPC $\left.{ }_{10}\right) 6$ and its components. (TPC 10 contains a $-\mathrm{O}-\mathrm{C}_{6} \mathrm{H}_{12}-\mathrm{O}-\mathrm{flexible}$ spacer and $\mathrm{M}$ contains a $-\mathrm{NH}-\mathrm{C}_{12} \mathrm{H}_{25}$ chain).

The supramolecular complex M6-(TPC 10$) 6$ was prepared by dissolving equimolar amounts of each pure component $\mathbf{M}$ and acid $\mathbf{T P C} \mathbf{C}_{10}$ in dichloromethane, which was evaporated at room 
temperature (ESI). $\mathrm{H}$-bonding interactions between $\mathbf{M}$ and acid $\mathrm{TPC}_{10}$ were analysed by ${ }^{1} \mathrm{H}$ NMR spectroscopy. The signals of the $\mathrm{N}-\mathrm{H}$ protons of $\mathbf{M}$ in $\mathrm{CD}_{2} \mathrm{Cl}_{2}$ solution, which overlapped between 4.60 and $4.90 \mathrm{ppm}$, were shifted to lower fields in the spectrum of the mixture of $\mathbf{M}$ with $\mathbf{T P C}_{10}$ in a 1:1 ratio. ${ }^{4} \mathrm{FTIR}$ studies allowed $\mathrm{H}$-bonding interactions to be observed in the bulk. The carbonyl stretching band of $\mathrm{TPC}_{10}$, which appears at $1687 \mathrm{~cm}^{-1}$ due to the cyclic dimeric form, shifted to $1679 \mathrm{~cm}^{-1}$ in the supramolecular complex (Figure S1 and S2, ESI).

M6-(TPC $\left.{ }_{10}\right) 6$ appeared as a homogeneous material when observed by polarized optical microscopy (POM) and the texture between crossed polarizers was consistent with a columnar mesophase (Figure S3, ESI). The transition to the isotropic liquid occurred at $73^{\circ} \mathrm{C}$ and this had an unusually high enthalpy $\left(44 \mathrm{~kJ} \mathrm{~mol}^{-1}\right)$, which indicates that the mesophase is highly organized. On cooling the material from the isotropic liquid the mesomorphic state was stable at room temperature, as deduced from the DSC thermogram, in which crystallization processes were not observed (Figure S3, ESI).

The X-ray diffraction patterns at room temperature contained a large number of reflections in the low angle region and this indicates a high degree of order (Figure 2a). These diffraction maxima correspond to distances with a ratio of $d, d / \sqrt{ } 4, d / \sqrt{ } 7, d / \sqrt{9}, d / \sqrt{13}$, etc., which are characteristic of a hexagonal lattice, and correspond to reflections (100), (200), (210), (300), (310), etc., of a hexagonal network with a lattice parameter $a=75.8 \AA$ (Table S1, ESI). In addition to the diffuse halo at $4.5 \AA$, which is characteristic of the liquid crystal state, a sharp and intense reflection at $3.5 \AA$ indicates long-range intracolumnar order. These lattice parameters are sufficiently high to support the formation of a rosette-like structure as a stacking unit and a calculated density value $\rho=0.88 \mathrm{~g} / \mathrm{cm}^{3}$ is obtained. ${ }^{4}$

The diffraction pattern in Figure $2 \mathrm{a}$ was essential for a proper determination of the lattice parameters since a large number of reflections were observed. However, the strongest reflections became saturated due to the long exposure time necessary for the weak reflections to be detected. Since these strong reflections are the most relevant ones for the construction of electron density maps, further measurements were carried out in the small angle region. The resulting diffraction diagram (Figure 2b) shows only three reflections, i.e., (100), (210), and (300), which are strong enough for an accurate determination of their relative intensities, and these were used for the calculation of the Fourier maps. The selected map is shown in Figure $2 \mathrm{c}$ and this represents variations in charge density relative to a mean value, which is the constant term in the Fourier series. The brightest regions are consistent with triphenylene units whereas the central dark circle corresponds to the empty interior of the rosette structure. The symmetry of the map corresponds to the plane group $p 6 \mathrm{~mm}$. This condition fixes the set of symmetry equivalent reflections that are necessary to construct the map. These reflections have the same intensity but appear overlapped in the diffraction diagram. 

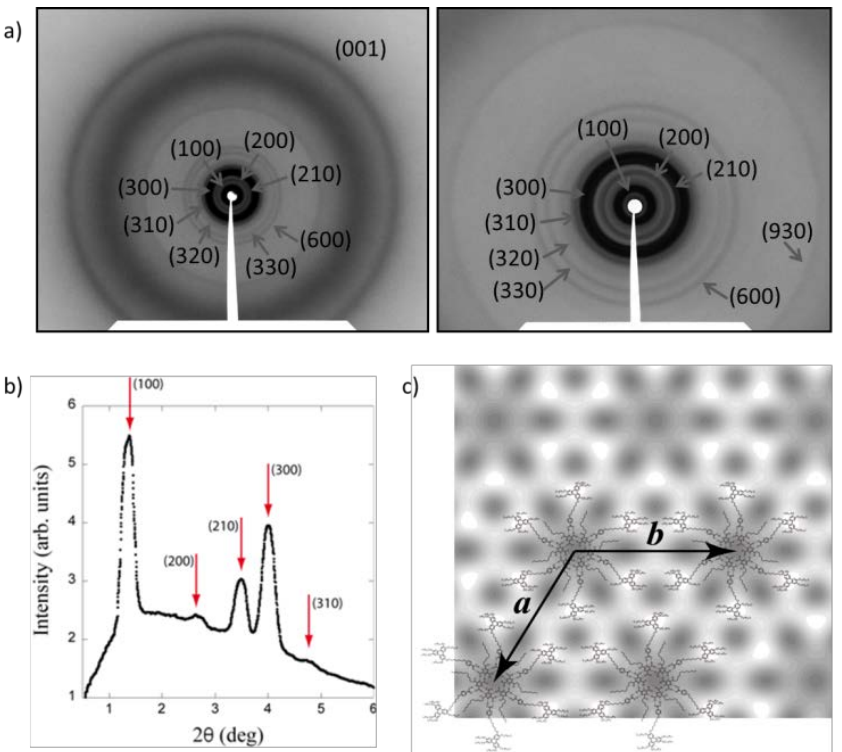

Figure 2. (a) Wide angle and small angle x-ray diffractograms of the mesophase of M6-(TPC $\mathbf{H}_{10} \mathbf{6}$ at room temperature. (b) Small angle diffraction diagram of $\mathbf{M 6}$ - $\left(\mathrm{TPC}_{10}\right) \mathbf{6}$ from which the relative intensities of the strongest reflections were determined. The diagram was obtained at room temperature after cooling the sample from the isotropic phase. (c) Electron density map calculated from the strong reflections in the diffraction pattern. The unit cell parameters and a scheme of the M6-(TPC $\left.\mathbf{~}_{10}\right) \mathbf{6}$ molecules are overprinted on the map. The areas of higher electronic density correspond to the triphenylene units.

The electrochemical properties of M6-(TPC 10$) 6$ were studied by cyclic voltammetry in thin film (Figure $\mathrm{S} 4, \mathrm{ESI})$. A HOMO energy value of $-5.4 \mathrm{eV}$ was calculated from the first oxidation wave $(1.07 \mathrm{~V})$. However, reduction waves were not observed in the potential window studied, i.e., between 0.0 and $-2.0 \mathrm{~V}$. The charge transport properties of M6-(TPC $\left.{ }_{10}\right) 6$ were measured using the Space-Charge-Limited Current (SCLC) method. A hole mobility of $4 \pm 0.7 \times 10^{-2} \mathrm{~cm}^{2} \mathrm{~s}^{-1} \mathrm{~V}^{-1}$ was determined and this value is two orders of magnitude higher than that measured for previous complexes containing the same triphenylene-containing benzoic acid. ${ }^{3}$ Indeed, this value is typical of triphenylene derivatives with a high degree of order. ${ }^{5}$ In contrast, it was not possible to measure electron mobility.

With the aim of shedding light on the intrinsic nature of M6-(TPC 10$) 6$ as a $p$ - or $n$-type organic semiconductor, a theoretical approach was employed that enabled us to interpret the charge mobility measurements. In this context, the DFT methodology is a powerful tool to establish structure-property relationships through a rigorous interpretation of the macroscopic properties of matter from an electronic perspective. DFT calculations were carried out on a model of the rosette -M6-(TPC 1$) 6$ - and the different parts composing the rosette -M6, TPC 1, M-TPC 1, M6-(pMBA)6in which long alkyl chains $\left(\mathrm{C}_{10}\right)$ bonded to melamine and triphenylene were replaced by methyl groups $\left(C_{1}\right)$ in order for the system to be computationally affordable (Figure 3$){ }^{6}$

From a theoretical point of view, the common strategy involved optimizing the molecular geometry and determining the basic charge injection and charge transport properties. Accordingly, M6-(TPC 1$) 6$ was optimized at the B3LYP/6-31G** level of theory, and the lengths of all possible $\mathrm{H}$-bonds were calculated and compared to those of different fragments (Figure 3 ), i.e., M6, M6-(pMBA)6, and M-TPC ${ }_{1}$. The optimized structure of M6-(TPC 1$) 6$ consists of a rosette of six melamine molecules connected to the adjacent rosette by a pair of $\mathrm{H}$-bonds $(\mathrm{N}-\mathrm{H} \cdots \mathrm{N})$ with lengths in the range 2.996-3.005 $\AA$, which is consistent with previously reported values. ${ }^{7}$ In addition, each triphenylene petal $\left(\mathrm{TPC}_{1}\right)$ is bonded to the melamine rosette by two $\mathrm{H}$-bonds established between the melamine and the carboxyl group of $\mathrm{TPC}_{1}$. The calculated $\mathrm{H}$-bond lengths are $2.564 \AA$ and $2.640 \AA$ for the $\mathrm{O}-\mathrm{H} \cdots \mathrm{N}$ and $\mathrm{O} \cdots \mathrm{H}-\mathrm{N}$ interactions, respectively (Table S2, ESI). 
The calculated electronic properties were the HOMO/LUMO energy levels, together with adiabatic ionization potentials, AIP, adiabatic electron affinities, AEA, and reorganization energy for both holes and electrons. Furthermore, the transfer integral of an M6-(TPC $)_{1} \mathbf{6}$ dimer was calculated considering the model proposed for the stacked complexes from XRD studies (Figure 2). Despite the fact that these electronic properties were calculated on an ideal, isolated complex, and neglecting thermal disorder, polarization effects of the neighbouring molecules, etc., they provide a reliable approximation to predict and interpret qualitatively, from an atomistic point of view, the $p$ - or $n$-type semiconductor behavior found in the experimental measurements. ${ }^{8}$ The HOMO and LUMO molecular orbitals for the studied structures are shown in Figure 3, and the calculated values for the electronic properties are collected in Table 1. These properties were also calculated for each individual component to estimate their influence in the final electronic properties of the complex.

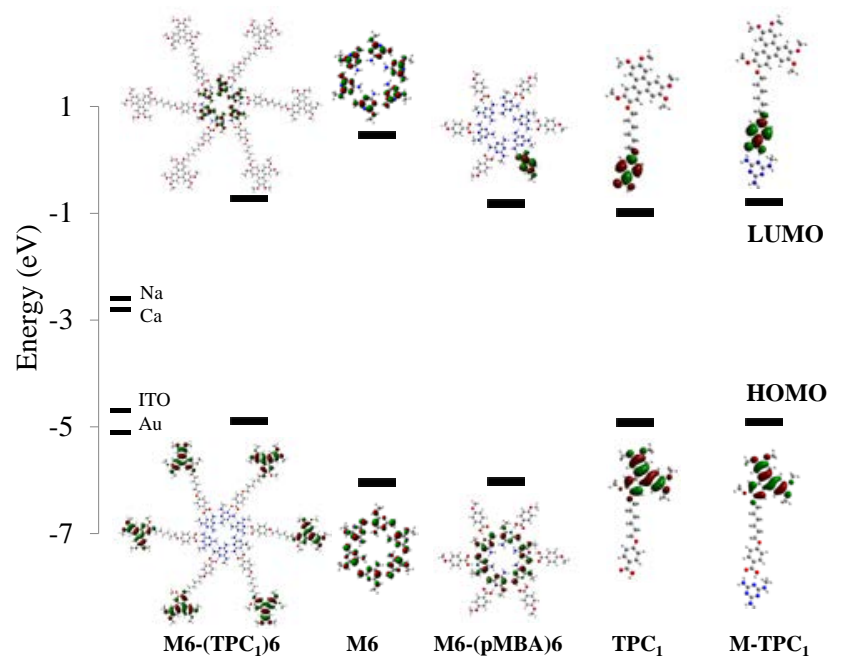

Figure 3. Theoretical HOMO and LUMO energy levels (in eV) calculated at the B3LYP/6-31G** level of theory. Illustration of the frontier molecular orbitals for the studied compounds calculated at the B3LYP/6-31G** level of theory. For M6-(TPC 1 )6, HOMO, H-1, H-2, H-3, H4 and $\mathrm{H}-5$ and LUMO, L+1 and L+2 are represented. The work functions of some common electrodes are also included.

For triphenylene-containing structures, the HOMO is always localized over the triphenylene moiety. However, the location of the LUMO is different depending on the moiety studied. Interestingly, the LUMO is located on the benzoic acid in fragments M6-(pMBA)6, TPC ${ }_{1}$ and M(TPC ${ }_{1}$ ) but it falls in the melamine rosette when M6-(TPC $\left.{ }_{1}\right) 6$ is formed. The energy levels for the complex, shown in Figure S6, indicate that the HOMO to HOMO-5 molecular orbitals are degenerate and, as a consequence, the HOMO should be composed of a combination of the lobes over the six triphenylene platforms (i.e., HOMO to HOMO-5). In the case of the LUMO, the degeneration of LUMO, LUMO+1 and LUMO+2 is less evident (Figure S6, ESI).

For an efficient charge injection, an ohmic contact is necessary between the work function of the electrode and the HOMO and LUMO energy levels, i.e., Ф-Еномо/Lumo $\leq 0.3 \mathrm{eV} .{ }^{9}$ As shown in Figure 3, the melamine rosette $\mathbf{M 6}$ has a relatively high LUMO energy level $(0.462 \mathrm{eV})$, which is largely stabilized when $\mathrm{H}$-bonds are formed between the carboxyl groups and the rosette, in M6-(pMBA)6 (-0.814) and M6-(TPC 1$) 6(-0.712)$. This is beneficial to the ambient stability of the material and also to electron injection. However, the LUMO energy level of complex M6-(TPC $\left.{ }_{1}\right) 6$ is still far from the work function of typical metals used as cathodes, such as $\mathrm{Na}(\Phi=-2.6 \mathrm{eV})$ and $\mathrm{Ca}(\Phi=-2.8 \mathrm{eV})$ and in this case electron injection should not be favored. The high energy level predicted for the LUMO in the complex could also explain the absence of a reduction process in the cyclic voltammetry measurements. 
Table 1. HOMO/LUMO energy levels, internal reorganization energies for holes $\left(\lambda_{+}\right)$and electrons $\left(\lambda_{-}\right)$, adiabatic ionization potential (AIP) and adiabatic electron affinity (AEA) (in eV) calculated at the B3LYP/3-21G* level of theory for M6-(TPC 1 )6, and at the B3LYP/6-31G** level of theory for the fragments.

\begin{tabular}{lrrrrrrr} 
Compound & HOMO LUMO & $\Delta \mathrm{E}_{\mathrm{H}-\mathrm{L}}$ & $\lambda_{+}$ & $\boldsymbol{\lambda}$ & AIP & AEA \\
\hline M6 & -6.047 & 0.462 & 6.507 & 0.069 & 0.366 & 6.869 & -1.158 \\
M6-(pMBA)6 & -6.029 & -0.814 & 5.215 & & & & \\
TPC $_{1}$ & -4.925 & -0.982 & 3.943 & 0.333 & 0.261 & 6.044 & -0.162 \\
M-TPC $_{1}$ & -4.913 & -0.796 & 4.117 & 0.272 & 0.407 & 5.996 & -0.150 \\
M6-(TPC $\left._{1}\right) 6$ & -4.897 & -0.712 & 4.185 & 0.058 & 0.056 & 5.033 & 0.285 \\
\hline
\end{tabular}

The calculated HOMO energy level of complex M6-(TPC $\left.{ }_{1}\right) 6$ is close to the work function of typical metals used as anodes, such as ITO $(\Phi=-4.7 \mathrm{eV})$ and $\mathrm{Au}(\Phi$ between -5.1 and -5.4 $\mathrm{eV}$ ), thus allowing an efficient hole injection. As shown in Figure 3, the presence of triphenylene in M6-(TPC $\left.{ }_{1}\right) 6$ significantly destabilizes the HOMO of the melamine rosettes, M6 and M6(pMBA)6, which reach energy levels close to the ITO and Au work functions.

For efficient hole and electron injection from the electrodes, it is necessary to have low AIP and high AEA values, respectively. The AIP values agree with those calculated for typical $p$ type organic semiconductors at the same level of theory, ${ }^{10}$ while the AEA values are far from the threshold value, ${ }^{11} 3 \mathrm{eV}$, so that electron injection is not predicted to be favored.

As a result, our theoretical calculations indicate that one of the explanations as to why the electron mobility could not be measured, although a high hole mobility is obtained, is that the calculated complex HOMO and AIP energy values are suitable to achieve efficient hole injection from common metal electrodes used as anodes, while electron injection is not efficient.

Charge transport within a discotic phase mainly occurs through a hopping regime and the rate of charge hopping $k$ can be estimated on the basis of semiclassical Marcus theory. ${ }^{12}$ According to the Marcus equation (ESI), two parameters must be optimized to achieve high charge mobilities: the reorganization energy $\left(\lambda_{+}\right.$and $\left.\lambda_{-}\right)$and the transfer integral. For the melamine rosette M6 $\lambda_{+} \ll<\lambda_{-}$and hole mobility should therefore be much more favored than electron mobility. When triphenylene-containing acids, $\mathbf{T P C}_{1}$, are incorporated to form complex M6$\left(\mathrm{TPC}_{1}\right) \mathbf{6}$, both hole and electron reorganization energies have very low and similar values $(0.06$ $\mathrm{eV}$ ), and this favors both hole and electron mobilities. This situation is in contrast to acid TPC $_{1}$ and its dimeric complex with melamine, $\mathbf{M}-\mathbf{T P C} \mathbf{C}_{\mathbf{1}}$, which show very different reorganization energy values for electrons and holes.

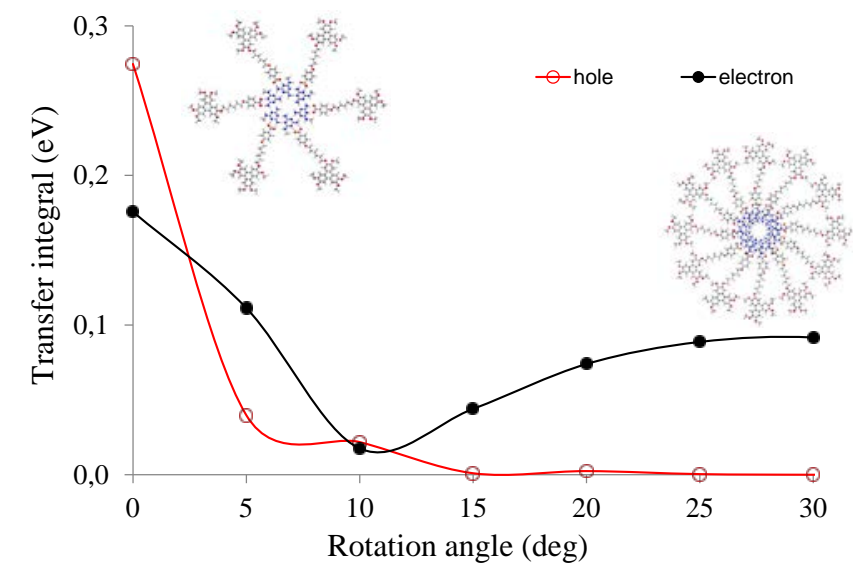

Figure. 4. Calculated hole $\left(t_{+}\right)$and electron ( $\left.t_{-}\right)$transfer integrals (in eV) as a function of rotation of one complex around the stacking axis (the interdisk distance of $3.5 \AA$ was fixed) calculated at the B3LYP/3-21G* level of theory.

In order to clarify the influence of the relative disposition of the disks on the transfer integral, the evolution of hole and electron transfer integrals was evaluated for a dimer by varying the 
rotation angle from $0^{\circ}$ to $30^{\circ}$ at intervals of $5^{\circ}$ (Figure 4). As expected, the highest values for the transfer integral were achieved in the cofacial orientation, when the molecular orbital overlap is maximum, and the hole transfer integral was higher than the electron one. Both $t_{+}$and $t_{-}$have theoretical values of 0.3 and $0.2 \mathrm{eV}$, respectively, and these are of the same order of magnitude as those determined for common organic semiconductors. ${ }^{13,14}$ However, $t_{+}$decreases dramatically upon changing from $0^{\circ}$ to $30^{\circ}$ as the HOMO is located over the triphenylene petal and the molecular orbital overlap is completely cancelled at $30^{\circ}$. In contrast, $\mathrm{t}$ - achieves another maximum at $30^{\circ}$ due to the fact that the LUMO overlap is not completely cancelled when the rosettes are rotated by $\leq 30^{\circ}$ (Figure 4), which means that a staggered arrangement would permit electron transport but impede hole transport.

In summary, the hierarchical implementation of $\mathrm{H}$-bonding and $\mathrm{m}$-stacking interactions, which lead to the formation of melamine rosettes linked to triphenylene-containing acids, has provided a well-organized semiconducting liquid crystal material with a hole mobility of $4 \pm 0.7 \times$ $10^{-2} \mathrm{~cm}^{2} \mathrm{~s}^{-1} \mathrm{~V}^{-1}$. As deduced from $X$-ray diffraction studies, segregated stacking of triphenylenes and melamine-rosettes occurs within the hexagonal columnar mesophase, and this is the optimum arrangement for charge-carrier mobility according to theoretical calculations. Indeed, DFT calculations have validated both the supramolecular rosette structure of the stacking units and provided necessary data to interpret charge transport properties. In particular, the calculation of molecular orbitals has not only confirmed that HOMO is located in triphenylenes, which was expectable, but also that LUMO is located over the melamine rosette (and not over the benzoic acid moiety), which is a less predictable result. This leads to an ideal double wire for charge transport with a hole inside the structure.

This is a new example of how the symbiosis between the experimental molecular design and theoretical calculations may lead to the achievement of functional, i.e., semiconducting, materials in which molecular and electronic architectures are decisive for the manifestation of the final properties.

\section{Acknowledgements}

This work was financially supported by the MINECO-FEDER funds (projects MAT2015-66208C3-1-P, CTQ2015-70174-P), the Gobierno de Aragón-FSE (E47_17R and B.F grant), the Consejería de Economía y Conocimiento, Junta de Andalucía (FQM-337) and the Universidad de Jaén (Acción 1_PIUJA 2017-18). AG thanks support from the ELIOTROPO project (PON03PE_00092_2). RT was supported by the MERAVIGLIE project (CUP J28C17000080006). The authors would like to thank the Centro de Servicios de Informática y Redes de Comunicaciones (CSIRC), Universidad de Granada, for providing the computing time and the Servicio General de Apoyo a la Investigación-SAI and CEQMA, Universidad de Zaragoza-CSIC.

Keywords: self-assembly $\bullet$ hydrogen bonds $\bullet$ liquid crystals $\bullet$ DFT $\bullet$ charge transport

[1] Recent reviews: (a) T. Kato, J. Uchida, T. Ichikawa and T. Sakamoto, Angew. Chem. Int. Ed., 2018, 57, 4355-4371. (b) T. Wöhrle, I. Wurzbach, J. Kirres, A. Kostidou, N. Kapernaum, J. Litterscheidt, J. C. Haenle, P. Staffeld, A. Baro, F. Giesselmann, S. Laschat, Chem. Rev., 2016, 116, 1139; (c) E.-K. Fleischmann, R. Zentel, Angew. Chem. Int. Ed., 2013, 52, 8810.

[2] Selected examples: (a) S. Bag, V. Maingi, P. K. Maiti, J. Yelk, M. A. Glaser, D. M. Walba, N. A. Clark, J. Chem. Phys., 2015, 143, 144505. (b) L. F. Dössel, V. Kamm, I. A. Howard, F. Laquai, W. Pisula, X. Feng, C. Li, M. Takase, T. Kudernac, S. De Feyter, K. Müllen, J. Am. Chem. Soc., 2012, 134, 5876. (c) J. M. Mativetsky, M. Kastler, R. C. Savage, D. Gentilini, M. Palma, W. Pisula, K. Müllen, P. Samorì, Adv. Funct. Mater., 2009, 19, 2486. (d) T. Yasuda, T. Shimizu, F. Liu, G. Ungar, T. Kato, J. Am. Chem. Soc., 2011, 133, 13437. (e) Y. Xiao, X. Su, L. Sosa-Vargas, E. Lacaze, B. Heinrich, B. Donnio, D. Kreher, F. Mathevet, A.- 
J. Attias, CrystEngComm, 2016, 18, 4787; (f) K.-Q. Zhao, L.-L. An, X.-B. Zhang, W.-H. Yu, P. Hu, B.-Q. Wang, J. Xu, Q.-D. Zeng, H. Monobe, Y. Shimizu, B. Heinrich, B. Donnio, Chem.-Eur. J., 2015, 21, 10379. g) M. Philipp, G. Matthias, L. Matthias, Chem.-Eur. J., 2017, 23, 1018.

[3] B. Feringán, P. Romero, J. L. Serrano, C. L. Folcia, J. Etxebarria, J. Ortega, R. Termine, A. Golemme, R. Giménez, T. Sierra, J. Am. Chem. Soc., 2016, 138, 12511.

[4] F. Vera, J. Barberá, P. Romero, José L. Serrano, M. B. Ros, T. Sierra, Angew. Chem. Int. Ed., 2010, 49, 4910-4914.

[5] (a) J. Simmerer, B. Glusen, W. Paulus, A. Kettner, P. Schuhmacher, D. Adam, K. H. Etzbach, K. Siemensmeyer, J. H. Wendorff, H. Ringsdorf, D. Haarer, Adv. Mater., 1996, 8, 815; (b) Y.-F. Wang, C.-X. Zhang, H. Wu, A. Zhang, J.-C. Wang, S.-F. Zhang, J.-L. Pu, Soft Matter, 2015, 11, 712; (c) J. Wang, C. Zhang, S. Zhang, X. Hao, F. Hong, A. Zhang, Y.-F. Wang, H. Wu, W. Zhang, J.-L. Pu, Liq. Cryst., 2017, 44, 394.

[6] S.M. Said, M.S. Mahmood, M.N. Daud, M.F. Mohd Sabri, N.A. Sairi, Liq. Cryst. 2016, 43, 2092.

[7] L. Vella-Zarb, D. Braga, A. G. Orpend, U. Baisch, CrystEngComm, 2014, 16, 8147.

[8] D. Khanh, K. R. Mahesh, W. Tonghui, J.L. Brédas, Chem. Mater. 2017, 29, 346.

[9] V. Coropceanu, J. Cornil, D. A. da Silva Filho, Y. Olivier, R. Silbey, J.-L. Brédas, Chem. Rev. 2007, 107, 926.

[10] M. C. Ruiz Delgado, K. R. Pigg, D. A. da Silva Filho, N. E. Gruhn, Y.Sakamoto, T. Suzuki, R. Malave Osuna, J. Casado, V. Hernández, J.T. López Navarrete, N.G. Martinelli, J. Cornil, R. S. Sánchez-Carrera, V. Coropceanu, J.-L. Brédas, J. Am. Chem. Soc. 2009, 131, 1502.

[11] Y. C. Chang, M.-Y. Kuo, C.-P. Chen, H.-F. Lu, I. Chao, J. Phys. Chem. C 2010, 114, 11595.

[12] R. A. Marcus, Rev. Mod. Phys. 1993, 65, 599.

[13] V. Lemaur, D. A. da Silva Filho, V. Coropceanu, M. Lehmann, Y. Geerts, J. Piris, M. G. Debije, A. M. van de Craats, K. Senthilkumar, L.D.A. Siebbeles, J.M. Warman, J.-L. Brédas, J. Cornil, J. Am. Chem. Soc. 2004, 126, 3271.

[14] X. Feng, V. Marcon, W. Pisula, M.R. Hansen, J. Kirkpatrick, F. Grozema, D. Andrienko, K. Kremer, K. Müllen, Nat. Mater. 2009, 8, 421. 